\title{
Optimization Fusion Approach for Image Segmentation using K-Means Algorithm
}

\author{
S.Mary Praveena \\ Research Scholar, EEE Department \\ PSG College of Technology \\ Coimbatore 641004 \\ Tamil nadu, India.
}

\author{
Dr.IlaVennila \\ Supervisor, EEE Department \\ PSG College Of Technology \\ Coimbatore 641004 \\ Tamil nadu, India.
}

\begin{abstract}
This paper presents a new, simple and Efficient segmentation approach,based on a fusion procedure which aims at combining several segmentation maps associated to simpler partition models in order to finally get a more reliable,accurate and a non-overlapped image result.The main objective of the paper is to get a non-overlapping and a reliable output by using k-means and genetic algorithm. The different colorspaces are to be fused in our application by the simple ( $K$-means based) clustering technique on an input image. The optimized range for $k$-means clustering values is obtained by performing genetic algorithm. Image segmentation for six color spaces are performed by $\mathrm{k}$ means. The k-means algorithm is an iterative technique that is used to partition an image into $K$ clusters. The obtained output remains simple to implement, fast, general enough to be applied to various computer vision applications (e.g., motion detection and segmentation). The result aims at developing an accurate and more reliable image which can be used in locating tumors, measure tissue volume, face recognition, finger print recognition and in locating an object clearly from a satellite image and in more.
\end{abstract}

Keywords: Genetic Algorithm, Segmentation, kmeans Algorithm, Optimization.

\section{1 .INTRODUCTION}

Image segmentation is a classic inverse problem which consists of achieving a compact region based description of the image scene by decomposing it into meaningful or spatially coherent regions sharing similar attributes. This low-level vision task is often the preliminary (and also crucial) step in many video and computer vision applications, such as object localization or recognition, data compression, tracking, image retrieval, or understanding. Because of its simplicity and efficiency, clustering approaches were one of the first techniques used for the segmentation of (textured) natural images [1]. After the selection and the extraction of the image features [usually based on color and/or texture and computed on (possibly) overlapping small windows centered around the pixel to be classified],the feature samples, handled as vectors, are grouped together in compact but well-separated clusters corresponding to each class of the image. The set of connected pixels belonging to each estimated class thus defined the different regions of the scene. The method known as k-means[3] (or Lloyd's algorithm).

\section{COLOR SPACES}

Color is extremely subjective and personal.to try to attribute numbers to the brains reaction to visual stimuli is very difficult.the aim of color spaces is to aid the process of describing color, either between people machines or programs. Color is the brains reaction to a specific visual stimulus.the color can be described by measuring its spectral power distribution this leads to large degree of redundancy.the reason for this redundancy is the eye's retina samples color using three broad bands, corresponding to red green and blue lights.the signals from these color sensitive cells (cones),together with those from the rods (sensitive to intensity only), are combined in the brain to give several different "sensation" of the color.these sensations have been defined by the CIE.

\section{3 .IMAGE SEGMENTATION}

Segmentation [1] refers to the process of partitioning a digital image into multiple regions (sets of pixels). The goal of segmentation is to simplify and change the representation of an image into something that is more meaningful and easier to analyze. Image segmentation is typically used to locate objects and boundaries (lines, curves, etc.) in images. The result of image segmentation is a set of regions that collectively cover the entire image, or a set of contours extracted from the image. Each of the pixels in a region are similar with respect to some characteristic or computed property, such as color, intensity, or texture[5][6]. Adjacent regions are significantly different with respect to the same characteristic(s). Segmentation is mainly used in medical imaging, Face recognition, Fingerprint recognition, Traffic control systems, Brake light detection, and Machine vision.

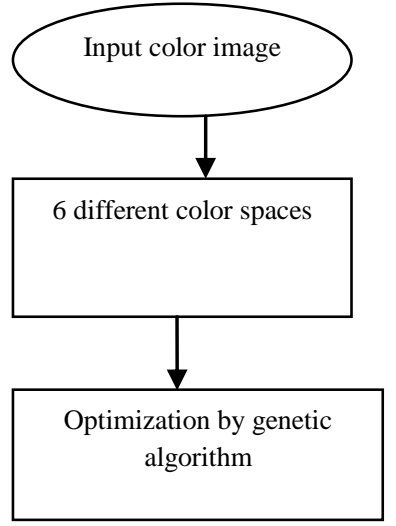




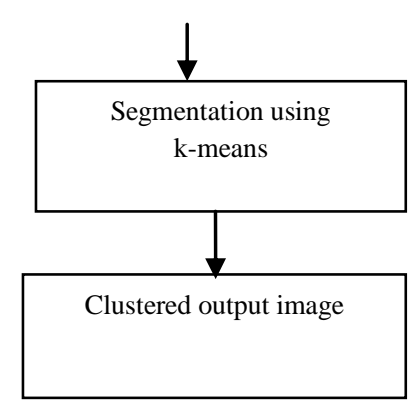

Fig 1:Flow chart for obtaining clustered image

\section{4 .COLOR SPACE CONVERSION}

Color is the brains reaction to a specific visual stimulus. Color can be described by measuring its spectral power distribution [11][17](the intensity of the visible electromagnetic radiation at many discrete wavelengths) this leads to a large degree of redundancy. The reason for this redundancy is that the eye's retina samples color using only three broad bands, roughly corresponding to red, green and blue light. The signals from these color sensitive cells (cones), together with those from the rods (sensitive to intensity only), are combined in the brain to give several different "sensations" of the color.

\subsection{DIFFERENT COLOR SPACES}

The image first considered is a color image; color image is taken because this image is further divided into different color spaces. Different colors provide different properties in special. A color image gives detailed information about the image and an attractive way of producing an image. Thus a color image is considered, it can a JPEG, BMP image. The color space may be device dependent and device independent,according to CIE standard six color spaces namely rgb,yiq,xyz,hsv,luv,lab are considered.

\subsubsection{RGB (Red Green Blue)}

This is an additive color system based on tri-chromatic theory. RGB is easy to implement but non-linear with visual perception. It is device dependent and specification of colors is semi-intuitive. RGB is very common, being used in virtually every computer system as well as television, video etc.

\subsubsection{Hue Saturation Value (Travis)}

These are the RGB-HSV conversions given by Travis. To convert from RGB to HSV (assuming normalized RGB values) first find the maximum and minimum values from the RGB triplet.

$\mathrm{s}=(\max -\min ) / \max$ and Value is $\mathrm{V}=\max$

The Hue, $\mathrm{H}$ is then calculated as follows. First calculate $\mathrm{R}^{\prime} \mathrm{G}^{\prime} \mathrm{B}$ '. Conditions is given by,

$$
\begin{aligned}
& \mathrm{R}^{\prime}=(\max -\mathrm{R}) /(\max -\min ) \\
& \mathrm{G}^{\prime}=(\max -\mathrm{G}) /(\max -\min ) \\
& \mathrm{B}^{\prime}=(\max -\mathrm{B}) /(\max -\min )
\end{aligned}
$$

Hue, $\mathrm{H}$, is then converted to degrees by multiplying by 60 giving HSV[18] with $\mathrm{S}$ and $\mathrm{V}$ between 0 and 1 and $\mathrm{H}$ between 0 and 360 .

\subsubsection{Y'I'Q' Color Space}

$\mathrm{Y}^{\prime}$ is similar to perceived luminance, I' and Q' carry color information and some luminance information and are derived by rotating the U'V' vector formed by color coding .The Y' signal usually has $4.2 \mathrm{MHz}$ bandwidth in a 525 line system. Originally the I' and Q' signals were to have different bandwidths $(0.5$ and $1.5 \mathrm{MHz})$ but they now commonly have the same bandwidth $(1 \mathrm{MHz})$. The system white point is Illuminant $\mathrm{C}$, the chromaticity co-ordinates are:-

$$
\begin{array}{ll}
\text { R: } x r=0.67 & y r=0.33 \\
\text { G: } x g=0.21 & y g=0.71 \\
\text { B: } x b=0.14 & y b=0.08
\end{array}
$$

white: $\mathrm{xn}=0.310063 ; \mathrm{yn}=0.316158$

\subsubsection{XYZ Color Space}

Conversion of RGB image pixel values to the CIE XYZ tristimulus values of the color displayed on the CRT can be achieved using a two stage process. Calculate the relationship between input image pixel values and displayed intensity. This relationship is the transfer function, it is simplified to gamma. The transfer functions will usually differ for each channel so are best measured independently. The second stage is to transform between the displayed red, green and blue to the CIE[25] tri stimulus values. This is most easily performed using a matrix transform of the following form:

$$
\left|\begin{array}{l}
x \\
y \\
z
\end{array}\right|=\begin{array}{ccc}
x r & x g & x b \\
y r & y g & y b \\
z r & z g & z b
\end{array} *\left|\begin{array}{l}
r \\
g \\
b
\end{array}\right|
$$

Where $\mathrm{X}, \mathrm{Y}, \mathrm{Z}$ are the desired CIE tri-stimulus values, R, G,B are the displayed RGB values obtained from the transfer functions and the $3 \times 3$ matrix is the measured CIE tri-stimulus values for CRT.

\subsubsection{LAB Color Space}

This is based directly on CIE XYZ (1931) and is another attempt to linearise the perceptibility of unit vector color differences. Again, it is non-linear, and the conversions are still reversible. Coloring information [5] is referred to the color of the white point of the system, subscript $n$. The nonlinear relationships for $\mathrm{L}^{*} \mathrm{a}^{*}$ and $\mathrm{b}^{*}$ are the same as for CIE LUV

$$
\begin{aligned}
& \mathrm{L}^{*}=\left\{116\left(\frac{y}{y n}\right) 1 / 3-16 \text { if } \frac{\mathrm{y}}{\mathrm{yn}}>0.008856\right. \\
& \mathrm{L}^{*}=\left\{903.3\left(\frac{y}{y n}\right) \text { if } \frac{\mathrm{y}}{\mathrm{yn}} \leq 0.008856\right.
\end{aligned}
$$

Again, L_ scales from 0 to 100.Again, there are polar parameters that more closely match the visual experience of colors.

$\mathrm{C}^{*}=\left(\mathrm{a}^{* 2}+\mathrm{b}^{* 2}\right)^{0.5} ; \mathrm{h}_{\mathrm{ab}}=\arctan \left(\mathrm{b} * / \mathrm{a}^{*}\right)$

Hue is an angle in four quadrants, and there is no saturation term in this system. When determining CIEL*a*b* or CIEL* $\mathrm{u}^{*} \mathrm{v} *$ values for CRT displayed colors it is usual to used the CRT's white point as the reference white. 


\subsection{6. $L * U * V *$ Color Space}

It is used to linearise the perceptibility of unit vector color differences. It is a non-linear color space, but the conversions are reversible. Coloring information is centered on the color of the white point of the system, subscript n, (D65 in most TV systems). The non-linear relationship for $\mathrm{Y}^{*}$ is intended to mimic the logarithmic response of the eye.

$$
\begin{gathered}
L^{*}=\left\{116\left(\frac{y}{y n}\right) 1 / 3-16 \quad \text { if } \frac{\mathrm{y}}{\mathrm{yn}}>0.008856\right. \\
\mathrm{L}^{*}=\left\{\begin{array}{c}
903.3\left(\frac{y}{y n}\right) \quad \text { if } \frac{\mathrm{y}}{\mathrm{yn}} \quad \leq 0.008856 \ldots . .(3) \\
\mathrm{u}^{*}=13\left(\mathrm{~L}^{*}\right)\left(\mathrm{U}^{\prime}-U n^{\prime}\right) \\
\mathrm{V}^{*}=13\left(\mathrm{~L}^{*}\right)\left(\mathrm{V}^{\prime}-V n^{\prime}\right)
\end{array}\right.
\end{gathered}
$$

\section{SEGMENTATION BY FUSION OF HISTOGRAMS}

Segmentation refers to the process of partitioning a digital image into multiple regions (sets of pixels). The goal of segmentation is to simplify and change the representation of an image into something that is more meaningful and easier to analyze. Image segmentation is typically used to locate objects and boundaries (lines, curves, etc.) in images. The result of image segmentation is a set of regions that collectively cover the entire image, or a set of contours extracted from the image. Each of the pixels in a region is similar with respect to some characteristic or computed property, such as color, intensity, or texture. Adjacent regions are significantly different with respect to the same characteristic(s).

Segmentation is mainly used in medical imaging, Face recognition, Fingerprint recognition, Traffic control systems, Brake light detection and Machine vision.

\subsection{Segmentation in Different Color Spaces}

The initial segmentation maps which will then be fused together by fusion framework are given, in this application, by a K-means[3][6] clustering technique, applied on an input image expressed by different color spaces, and using as simple cues (i.e., as input multidimensional feature descriptor) the set of values of the re-quantized color histogram (with equidistant binning) estimated around the pixel to be classified. In this application, this local histogram is equally re-quantized (for each of the three color channels) in a $\mathrm{N}_{\mathrm{b}}=5 * 5 * 5=125$ bins descriptor, computed on an overlapping squared fixed-size $\left(\mathrm{N}_{\mathrm{w}}=7\right)$ neighborhood centered around the pixel to be classified. This estimation can be quickly computed by using a more coarsely requantized color space and then computed the bin index that represents each re-quantized color

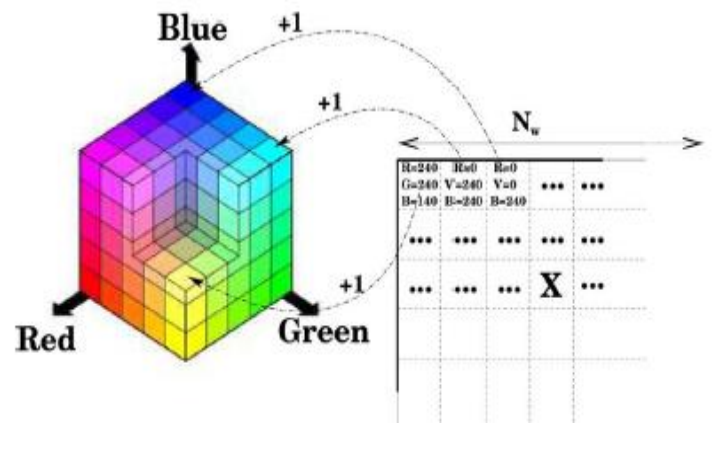

Fig 2:Estimation of each pixel $x$

Mathematically, let $b(x) €\left\{0, \ldots . N_{b}-1\right.$ denote the bin index associated with the color vector $\mathrm{y}(\mathrm{x})$ at pixel location (lying on a pixel grid) and $\mathrm{N}(\mathrm{x})$ be the set of pixel locations $\mathrm{X}$ within the squared neighborhood region (of fixed-size $\mathrm{N}_{\mathrm{w}} * \mathrm{~N}_{\mathrm{w}}$ ) centered at pixel location $\mathrm{X}$ (in which local color information will be gathered).An estimate of $\mathrm{h}(\mathrm{x})=\{\mathrm{h}(\mathrm{n} ; \mathrm{x})\}_{\mathrm{n}=0} \quad \ldots . \mathrm{Nb}-1 \quad$ of 125 bins descriptor, characterizing the color distribution[4] for each pixel to be classified, is given by the following standard bin counting procedure:

$$
\mathrm{h}(\mathrm{n} ; \mathrm{x})=\mathrm{k} \sum_{N e N(X)} \delta[\mathrm{b}(\mathrm{u})-\mathrm{n}]
$$

Where Kronecker delta function and is a $\mathrm{k}=1 /(\mathrm{Nw})^{2}$ normalization constant ensuring is a normalization constant ensuring $\quad \sum_{\mathrm{n}=0}$.Algorithm I. Estimation, for each pixel, of the bins descriptor. Estimation of the $\mathrm{N}_{\mathrm{b}}=\mathrm{q}^{3}$ bins descriptor $\mathrm{Nx}$ set of pixel locations $\mathrm{x}$ within the $\mathrm{Nw} * \mathrm{Nw}$ neighborhood region centered at $\mathrm{h}[\mathrm{B}$ Bins descriptor: Array of $\mathrm{Nb}$ floats $(\mathrm{h}[0], \mathrm{h}[1], \ldots . . . \mathrm{h}[\mathrm{Nb}-1])$. [.] Integer part For each pixel $\mathrm{x} \in \mathrm{Nx}$ with color value $\mathrm{Rx}, \mathrm{Gx}, \mathrm{Bx}$

$$
\begin{aligned}
& \mathrm{k} \leftarrow \mathrm{q}^{2} \cdot\left[\mathrm{q} \cdot \mathrm{R}_{\mathrm{x}} / 256\right]+\mathrm{q} \cdot\left[\mathrm{q} \cdot \mathrm{G}_{\mathrm{x}} / 256\right]+\left[\mathrm{q} \cdot \mathrm{B}_{\mathrm{x}} / 256\right] \\
& \mathrm{h}[\mathrm{k}] \leftarrow \mathrm{h}[\mathrm{k}]+1 /\left(\mathrm{N}_{\mathrm{w}}\right)^{2}
\end{aligned}
$$

Finally, these (125-bin) descriptors are grouped together into different clusters (corresponding to each class of the image) by the classical $\mathrm{K}$-means algorithm with the classical Euclidean distance. This simple segmentation strategy of the input image into $\mathrm{K}_{1}$ classes is repeated for different color spaces which can be viewed as different image channels provided by various sensors or captors (or as a multichannel filtering where the channels are represented by the different color spaces). In our application, we use $\mathrm{N}_{\mathrm{s}}$ segmentations provided by the $\mathrm{Ns}=6$ color spaces namely RGB,HSV,YIQ,XYZ,LAB,LUV color spaces.

\subsection{Fusion of Segmentation Map}

The key idea of the proposed fusion procedure simply consists of considering, for each site (or pixel to be classified), the local histogram of the class (or texton) labels of each segmentation to be fused, computed on a squared fixed-size neighborhood centered around the pixel, 
as input feature vector of a final clustering procedure.For a fusion of $\mathrm{N}_{\mathrm{s}}$ segmentation with $\mathrm{k}_{1}$ classes into a segmentation with $\mathrm{k}_{2}$ classes, the preliminary feature extraction step of this fusion procedure thus yields to $\mathrm{N}_{\mathrm{s}}\left(\mathrm{K}_{1}\right.$ -bin) histograms which are then gathered together in order to form, a $\mathrm{k}_{1} * \mathrm{~N}_{\mathrm{s}}$-dimensional feature vector or a final (K1 $* \mathrm{~N}_{\mathrm{s}}$ ) bin histogram which is then normalized to sum to one, so that it is also a probability distribution function. The proposed fusion procedure is then herein simply considered as a problem of clustering local histograms of (preliminary estimated) class labels computed around and associated to each site. To this end, we use, once again, a K-means clustering procedure exploiting, for this fusion step, an histogram-based similarity measure derived from the Bhattacharya similarity coefficient. Given a normalized histogram $\left\{\mathrm{h}(\mathrm{n} ; \mathrm{x})_{\mathrm{n}=0} \ldots . . \mathrm{Nb}-1=\mathrm{k} 1 \mathrm{~N}_{\mathrm{s}}-1\right.$ (at pixel location)and a reference histogram $\{\mathrm{h} *(\mathrm{n})\}_{\mathrm{n}=0 \ldots . \mathrm{Nb}-1}$ (representing one of the cluster centers of each class of a -means procedure), the Bhattacharya distance between these two histograms is given as,

$$
\mathrm{DB}\left[\mathrm{h}^{*}, \mathrm{~h}(\mathrm{x})\right]=\left(1-\sum_{n=0}^{N b-1} \sqrt{h *(n)} h(n ; x)\right)^{1 / 2}
$$

and a $\mathrm{K}$-means algorithm based on this distance converges in all tested examples. The pre estimated label fields to be fused along with the fusion procedure can be viewed (and qualitatively explained) as a two-step hierarchical segmentation procedure in which, first, a texton segmentation map[11] (in each color space) is estimated and, second, a final clustering, taking into account this mixture of textons (expressed in the set of color space ), is then used for a final clustering. We recall that a texton, in our framework, is defined by a nonparametric mixture of colors.A final merging step is necessary and is used to avoid over segmentation for some images. It consists of fusing each region (i.e., set of connected pixels belonging to the same class) of the resulting segmentation map with one of its neighboring region $\mathrm{R}$ if the distance $\mathrm{D}$ merging is below a given threshold $\mathrm{k}$ (or if its size is below 50 pixels with the closest region in the $\mathrm{D}_{\text {merging }}$ distance sense)

$$
\text { Dmerging }=x \min \in \mathrm{R}\{\mathrm{DB} \mid[\mathrm{h} 0(\mathrm{n}), \mathrm{h}+(\mathrm{n} ; \mathrm{x})]\}
$$

First summation is done on the six used color spaces, and $\mathrm{h}^{\mathrm{O}}(\mathrm{n})$ designates the normalized nonparametric histogram of the set of pixels belonging to the region to be merged and $\mathrm{h}^{\mathfrak{f}}(\mathrm{n} ; \mathrm{x})$ is the normalized histogram, computed on a squared fixed-size $\left(\mathrm{N}_{\mathrm{w}}\right)$ windows (at pixel location and totally included in the region). For this merging procedure, the two histograms are equally re-quantized (for each of the three color channels) with $4 * 4 * 4$ bins

\section{K-MEANS CLUSTERING}

K-means (MacQueen, 1967) is one of the simplest unsupervised learning algorithms that solve the well known clustering problem. The procedure follows a simple and easy way to classify a given data set through a certain number of clusters (assume $\mathrm{k}$ clusters) fixed a priori. The main idea is to define $\mathrm{k}$ centroids,[12] one for each cluster. These centroids should be placed in a cunning way because of different location causes different result. So, the better choice is to place them as much as possible far away from each other. The next step is to take each point belonging to a given data set and associate it to the nearest centroid. When no point is pending, the first step is completed and an early group age is done. At this point we need to recalculate k new centroids as barycenters[4] of the clusters resulting from the previous step. After we have these k new centroids, a new binding has to be done between the same data set points and the nearest new centroid. A loop has been generated. As a result of this loop we may notice that the $\mathrm{k}$ centroids change their location step by step until no more changes are done. In other words centroids do not moveanymore. K-Means clustering generates a specific number of disjoint, flat (non-hierarchical) clusters. It is well suited to generating global clusters. The K-Means method is numerical, unsupervised, non-deterministic and iterative.

\subsection{K-Means Properties}

- There are always K clusters.

- There is always at least one item in each cluster.

- The clusters are non-hierarchical and they do not overlap.

- Every member of a cluster is closer to its cluster than any other cluster because closeness does not always involve the 'center' of clusters.

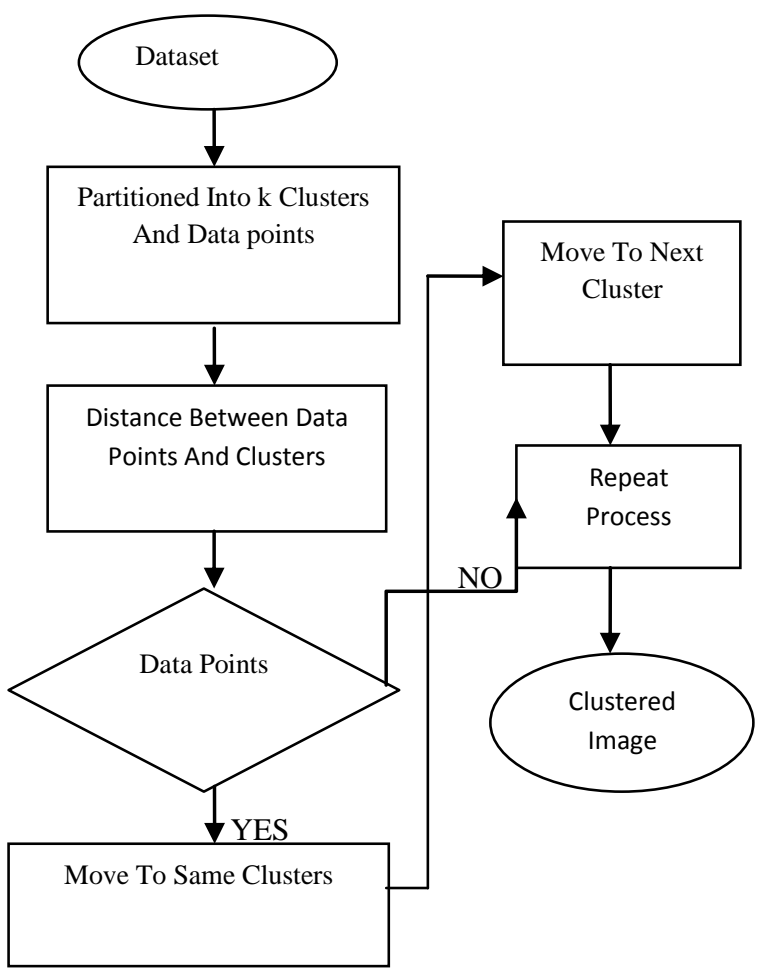

Fig 3: Flow chart for k-Means Algorithm

\subsection{Process of Genetic Algorithm}

Step 1:The algorithm usually starts from a population of randomly generated individuals and happens in generations. 
Step 2:In each generation, the fitness of every individual in the population is evaluated.

Step 3:Multiple individuals are randomly selected from the current population and modified to form a new population.

Step 4:The new population is then used in the next iteration of the algorithm.

Step 5:The algorithm terminates when either a maximum number of generations has been produced, or a satisfactory fitness level has been reached for the population.

Step 6:Genetic algorithms find application in bioinformatics, phylogenetics, engineering and manufacturing

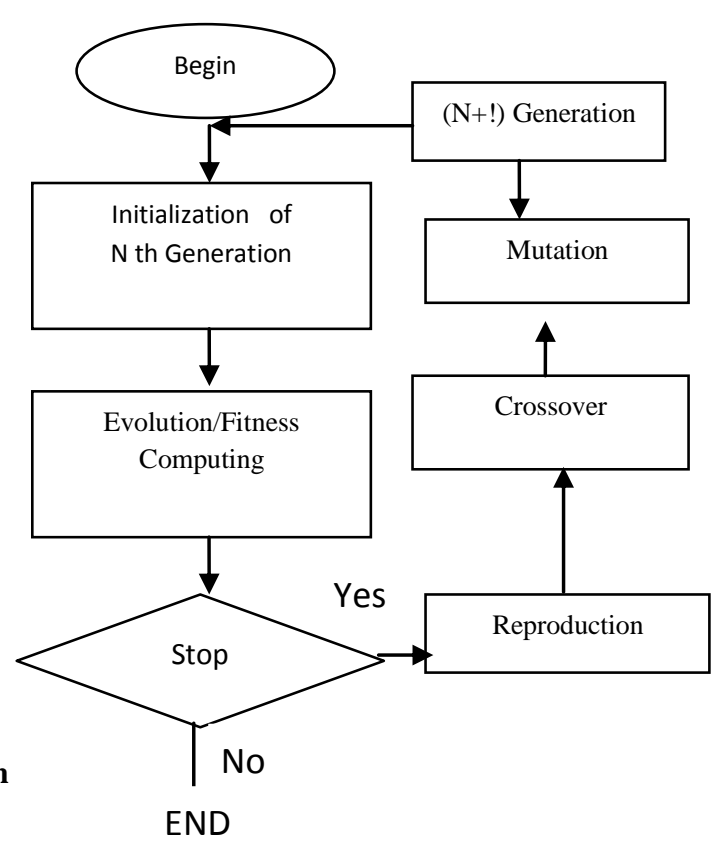

\subsection{Color Space Conversions}
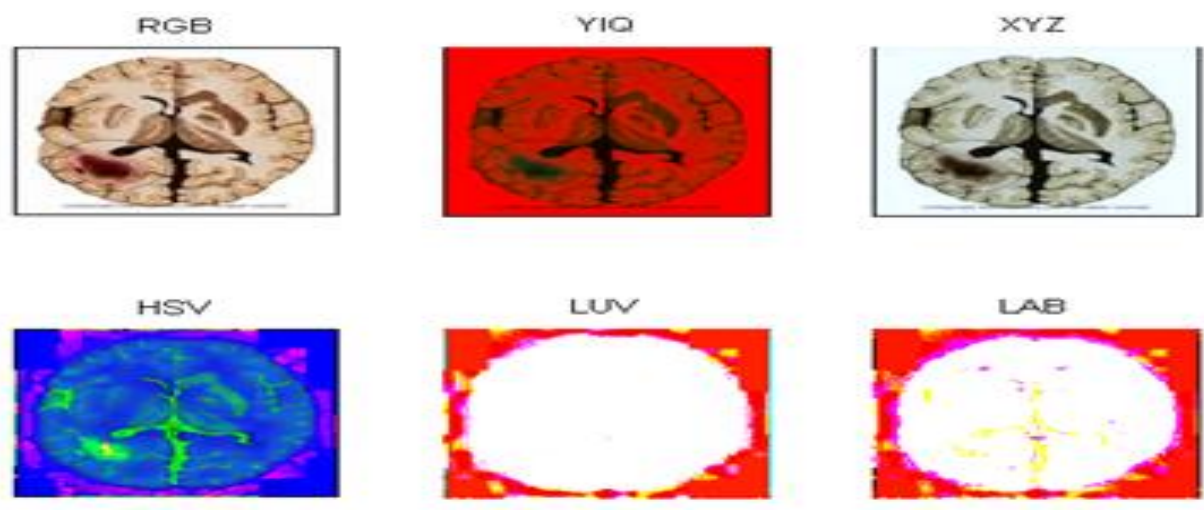

Fig 5: Diagram for Color Spaces

\subsection{Segmented Images Using $k$ Means}
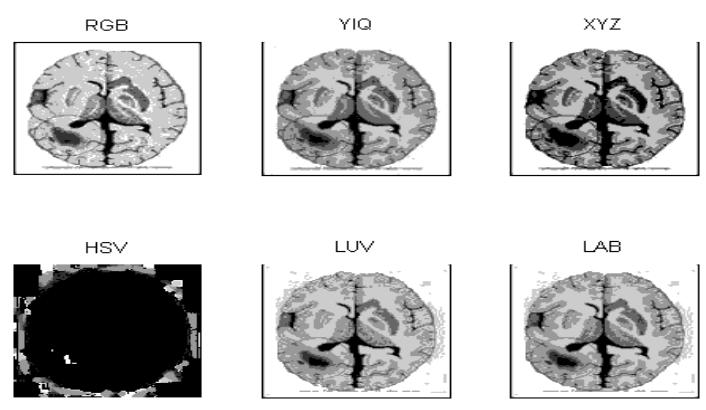

Fig 6:Segmented images using k-means 


\subsection{Fused Images}
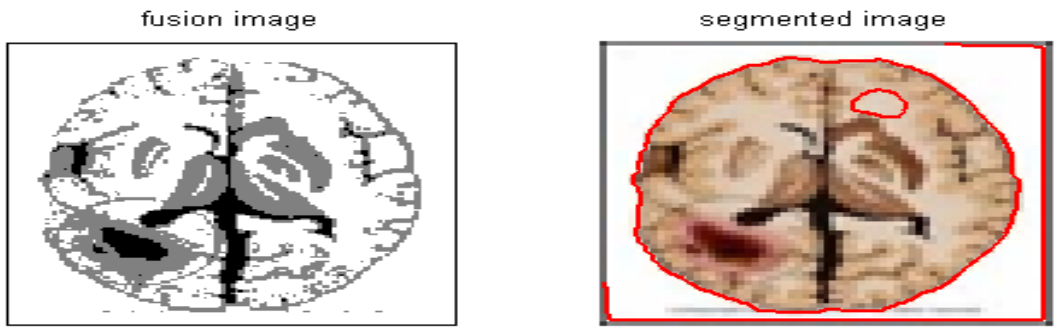

Fig 7:Fused Image Using K Means

\section{PERFORMANCE MEASURES}

TABLE 1: AVERAGE PERFORMANCE OF ALGORITHM FOR SEVERAL VALUES OF ITS INTERNAL PARAMETERS

\begin{tabular}{|c|c|c|c|c|}
\hline Parameter & Boundary & RI & VOI & GCE \\
\hline RGB(proposed) & 4.4713 & 0.66438 & 3.4926 & 0.49276 \\
\hline (existing) & 9.3065 & 0.80910 & 2.1282 & 0.17975 \\
\hline YIQ(proposed) & 4.5963 & 0.64427 & 3.3973 & 0.47207 \\
\hline (existing) & 11.1331 & 0.79055 & 2.2627 & 0.26257 \\
\hline XYZ(proposed) & 3.5852 & 0.69099 & 3.6899 & 0.56648 \\
\hline (existing) & 11.5585 & 0.80683 & 2.0082 & 0.25385 \\
\hline HSV(proposed) & 4.3259 & 0.68278 & 3.2855 & 0.47484 \\
\hline (existing) & 9.8310 & 0.79904 & 2.4031 & 0.16182 \\
\hline LUV(proposed) & 4.1055 & 0.61257 & 3.2714 & 0.42793 \\
\hline (existing) & 10.8777 & 0.74751 & 3.0254 & 0.33568 \\
\hline LAB(proposed) & 5.444 & 0.61751 & 3.2931 & 0.44131 \\
\hline (existing) & 9.3800 & 0.80549 & 2.4849 & 0.22742 \\
\hline FUSION(proposed) & 6.6523 & 0.57372 & 3.2766 & 0.4151 \\
\hline (existing) & 10.2006 & 0.83375 & 1.6808 & 0.13678 \\
\hline
\end{tabular}


Where

GCE- Global consistency Measure

BDE-Boundary Displacement Error

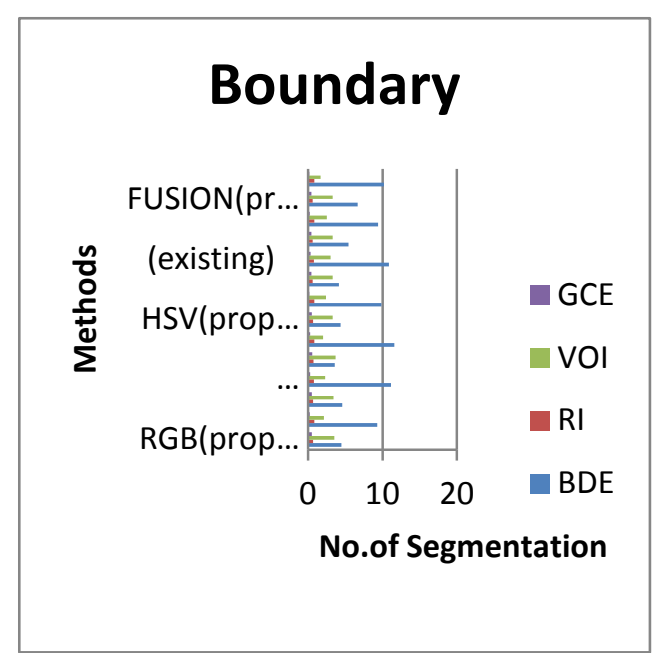

Fig:8a. characteristics of boundary values

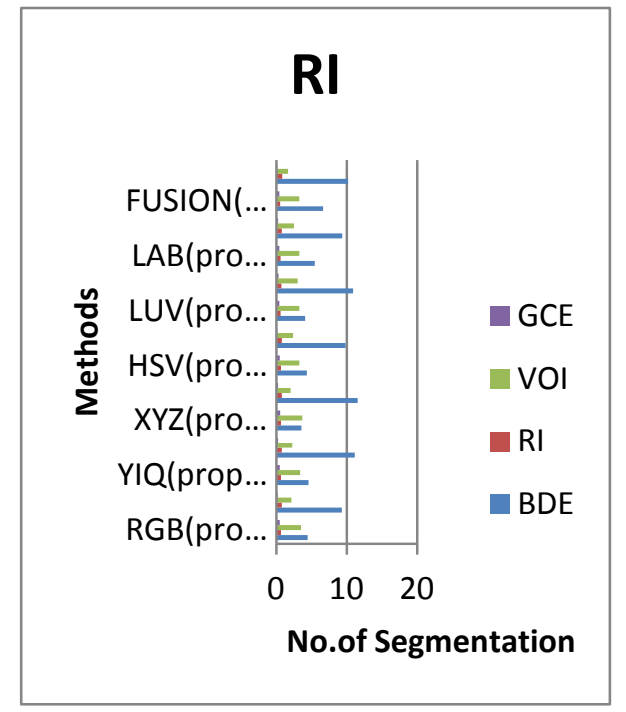

Fig:8c.c characteristics of RI values

\section{CONCLUSION}

A new segmented and optimized image using genetic algorithm is fused to get more reliable and accurate result. This fusion framework remains simple, fast, easily parallelizable, general enough to be applied to various computer vision applications, and performs competitively among the recently reported state-of-the-art segmentation methods. This novel scheme can be used in the image processing and computer vision community, and has

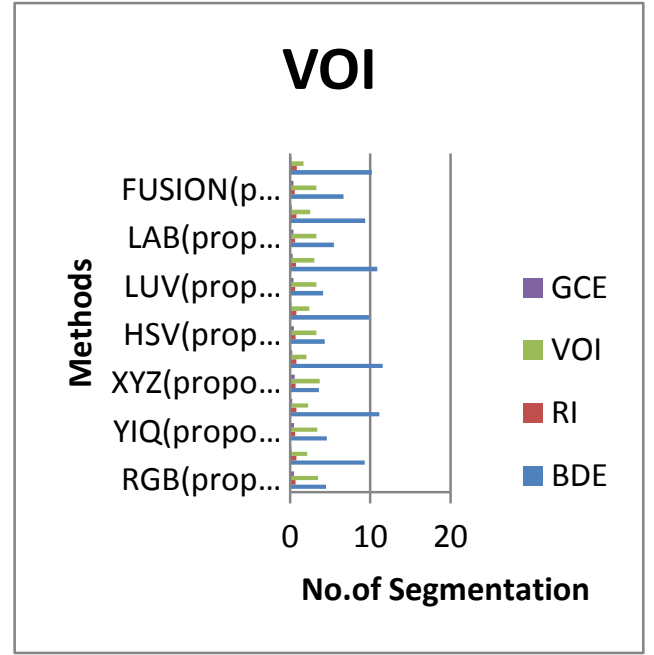

Fig:8b. Characteristics of VOI values

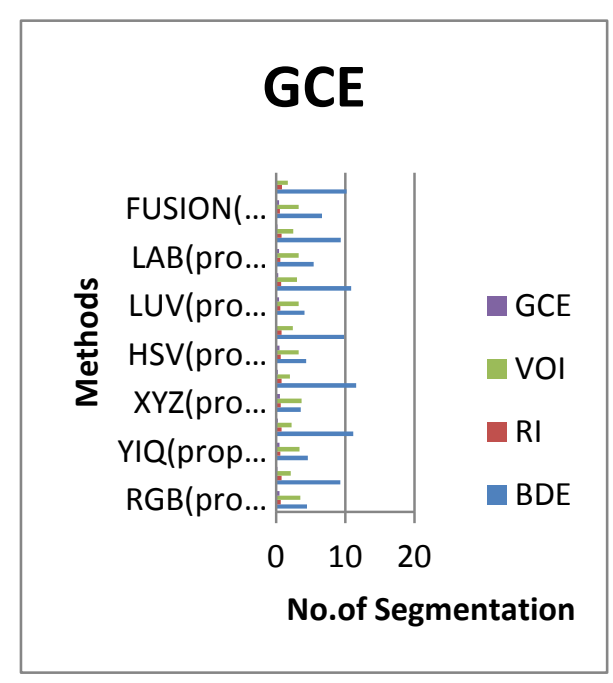

Fig:8c. characteristics of GCE values

widespread image coding, robotics, and human-computer interaction.

\section{REFERENCES}

[1] S. P. Lloyd, "Least squares quantization in PCM," IEEE Trans. Inf.Theory, vol. IT-28, no. 2, pp. 129-136, Mar. 1982. 
[2] D. Comaniciu and P. Meer, "Mean shift: A robust approach toward feature space analysis," IEEE Trans. Pattern Anal. Mach. Intell., vol.24, no. 5, pp. 603-619, May 2002.

[3] J. Shi and J. Malik, "Normalized cuts and image segmentation," IEEE Trans. Pattern Anal. Mach. Intell., vol. 22, no. 8, pp. 888-905, Aug.2000.

[4] S. Zhu and A. Yuille, "Region competition: Unifying snakes, region growing, and Bayes/MDL for multiband image segmentation," IEEE Trans. Pattern Anal. Mach. Intell., vol. 18, no. 9, pp. 884-900, Sep.1996.

[5] M. Mignotte, C. Collet, P. Pérez, and P. Bouthemy, "Sonar image segmentation using a hierarchical MRF model," IEEE Trans. ImageProcess., vol. 9, no. 7, pp. 1216-1231, Jul. 2000.

[6] F. Destrempes, J.-F. Angers, and M. Mignotte, "Fusion of hidden Markov random field models and its Bayesian estimation," IEEE Trans. Image Process., vol. 15, no. 10, pp. 2920-2935, Oct. 2006.

[7] J.-P. Braquelaire and L. Brun, "Comparison and optimization of methods of color image quantization," IEEE Trans. Image Process., vol. 6, no. 7, pp. 10481952, Jul. 1997.

[8] H. Stokman and T. Gevers, "Selection and fusion of color models for image feature detection," IEEE Trans. Pattern Anal. Mach. Intell., vol. 29, no. 3, pp. 371-381, Mar. 2007.

[9] R. Unnikrishnan, C. Pantofaru, and M. Hebert, "A measure for objective evaluation of image segmentation algorithms," in Proc. IEEE Conf. Computer Vision and Pattern Recognition Workshop on Empirical Evaluation Methods in Computer Vision, Jun. 2005, vol. 3, pp.34-41.

[10] S. Banks, Signal Processing, Image Processing and Pattern Recognition englewood Cliffs, NJ: PrenticeHall, 1990.

[11] P. Berkhin, "Survey of clustering data mining techniques," AccrueSoftware, San Jose, CA, 2002.

[12] J. Besag, "On the statistical analysis of dirty pictures," J. Roy. Statist.Soc. B, vol. 48, pp. 259-302, 1986.

[13] P. Felzenszwalb and D. Huttenlocher, "Efficient graphbased image segmentation," Int. J. Comput. Vis., vol. 59, pp. 167-181, 2004.

[14] M. Mignotte, C. Collet, P. Pérez, and P. Bouthemy, "Three-class Markovian segmentation of high resolution sonar images," Comput. Vis.Image Understand., vol. 76, no. 3, pp. 191-204, 1999.

[15] Z. Kato, T. C. Pong, and G. Q. Song, "Unsupervised segmentation of color textured images using a multilayer MRF model," in Proc. Int. Conf. Image Processing, Barcelona, Spain, Sep. 2003, pp. 961-964.

[16] P. Pérez, C. Hue, J. Vermaak, and M. Gangnet, "Colorbased probabilistic tracking," in Proc. Eur. Conf. Computer Vision, Copenhagen,Denmark, Jun. 2002, pp. 661-675.
[17] J. B. Martinkauppi, M. N. Soriano, and M. H. Laaksonen, "Behavior of skin color under varying illumination seen by different cameras at different color spaces," in Proc. SPIE, Machine Vision Applications in Industrial Inspection IX, San Jose, CA, Jan. 2001, pp. 102-113.

[18] Z. Kato, "A Markov random field image segmentation model for color textured images," Image Vis. Comput., vol. 24, no. 10, pp. 1103-1114,2006.

[19] E. Maggio and A. Cavallaro, "Multi-part target representation for color tracking," in Proc. Int. Conf. Image Processing, Italy, Genova, Sep.2005, pp. 729732

[20] M. Meila, "Comparing clusterings-An axiomatic view," in Proc. $22^{\text {nd }}$ Int. Conf. Machine Learning, 2005, pp. 577-584.

[21] D. Martin, C. Fowlkes, D. Tal, and J. Malik, "A database of human segmented natural images and its application to evaluating segmentationalgorithms and measuring ecological statistics," in Proc. 8th Int. Conf. Computer Vision, Jul. 2001, vol. 2, pp. 416-423.

[22] J. Freixenet, X. Munoz, D. Raba, J. Marti, and X. Cufi, "Yet another survey on image segmentation: Region and boundary information integration," in Proc. 7th Eur. Conf. Computer Vision Part III, Copenhagen,Denmark, May 2002, pp. 408-422, LNCS.

[23] A. Y. Yang, J. Wright, S. Sastry, and Y. Ma, "Unsupervised segmentation of natural images via lossy data compression," Comput. Vis. Image Understand., 2007, submitted for publication.

[24] Y. Ma, H. Derksen, W. Hong, and J. Wright, "Segmentation of multivariate mixed data via lossy coding and compression," IEEE Trans.Pattern Anal. Mach. Intell., vol. 29, no. 9, pp. 1546-1562, Sep. 2007.

[25] A. Y. Yang, J. Wright, S. Sastry, and Y. Ma, "Unsupervised segmentation of natural images via lossy data compression," Elect. Eng. Comput.Sci. Dept. Univ. California, Berkeley, 2006 [Online].Available:http://www.eecs.berkeley.edu/Pubs/ TechRpts/2006/EECS-2006-195.html.

\section{AUTHOR BIOGRAPHIES}

S.Mary Praveena, is pursuing $\mathrm{PhD}$ under the Guidance of Dr. Ila Vennila. Her research area are Digital Image Processing, Neural Networks, and Genetic Algorithm. Her publication includes several papers in National International conferences. She is a member of ISTE.

Dr. Ila.Vennila is working as an Assistant Professor in Electrical and Electronics Department, PSG college of Technology,Coimbatore.Her research interest includes Digital Signal Processing, Digital Image Processing .Dr.Vennila has over 23 years of Experience in teaching. Her publication includes 10 journal papers includes 10 journal papers and 30 National / International Conferences. 